http://dx.doi.org/10.21611/qirt.1992.024

\title{
Lockin vibrothermal inspection of polymer composites
}

\author{
by G. BUSSE, M. BAUER, W. RIPPEL and D. WU (*)
}

Institut für Kunststoffprüfung und Kunststoffkunde, Universität Stuttgart, Pfaffenwaldring 32,W-7000 Stuttgart 80, Germany.

\begin{abstract}
Vibrothermography uses thermographic equipment to image the average temperature distribution of oscillating components. In this paper we report on experiments where magnitude and phase of the temperature modulation generated by modulated stress were analysed. Investigations on various polymer composites reveal both the thermoelastic effect and loss angle induced energy dissipation as the mechanism for thermal wave generation.
\end{abstract}

\section{Introduction}

Polymer materials differ in many ways from metals. That is why their use increases in various applications. On the other hand, the different physical properties provide the background both for failure mechanisms and for methods to monitor them.

Some of the properties that need to be mentioned as a background to this paper are the strong acoustical damping related to a loss angle or hysteresis effect which exceeds the one of metals by orders of magnitude, the low thermal conductivity keeping heat localized to where it was generated, and high emissivity in the spectral range where thermal infrared black body radiation has its maximum at room temperature. That is why vibrothermography is so well suited to reveal sample areas where stress is concentrated or where hidden defects are located [1]. If the polymer component under inspection does not have a surface with a homogeneous emissivity, optical structure in the infrared will make interpretation of thermographic images more difficult.

It is known from thermal wave work that signal phase eliminates this kind of problems [2]. Results obtained recently for lockin thermography [3] also confirmed the advantage of phase images. The transfer of this experience to vibrothermography means that thermal waves are generated by application of periodical stress effects in the volume of polymer materials and that their phase with respect to mechanical excitation (instead of optical excitation used for photothermal work) provides information which may differ from conventional vibrothermography where only the dc-part of generated heat is analysed. The ac-part caused by stress induced thermal effects under oscillating load is the thermal wave generator which, in contrast to photothermal techniques, is not confined to the optical penetration depth. Hence, under the aspect of thermal waves, the situation corresponds to optically transparent samples under photothermal inspection. Table 1 shows how the kind of heat source and its temporal behaviour is related to the various existing techniques.

The common background is that the modulated thermal effect of a modulated input acting on a loss mechanism is analysed. The term "lockin thermography" would describe all these effects (depending on the kind of excitation) if the modulation of the temperature field were to be analysed using thermographic equipment. Correspondingly the term lockin radiometry would be useful in a situation where only one spot is observed, like in the lockin vibrothermal radiometry described in the following. 


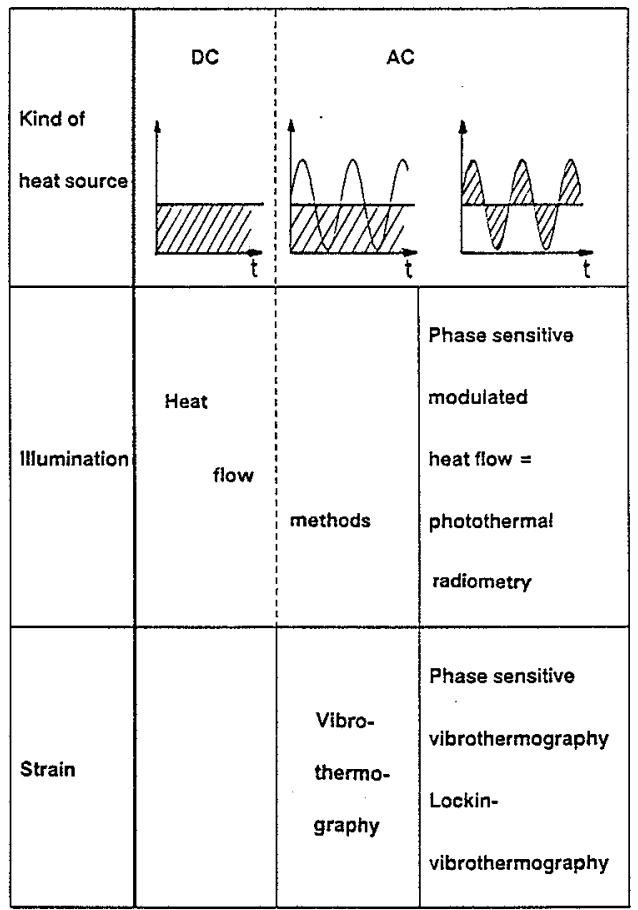

Table 1 - Various existing techniques related to temporal behaviour of heat source.

Conventional heat-flow methods and vibrothermography depend only on the average value of modulated $(A C)$ heat sources.

\section{Experimental arrangement}

The basic setup is shown in figure 1. The sample was exposed to an oscillating load while an infrared detector with a lens in front of it observed a $1 \mathrm{~mm}$ diameter spot on the sample. The detector was located on a scanning unit to allow for analysis of local variations. This setup is partly similar to the SPATE-device where the thermoelastic effect is used to monitor stress fields or thickness distribution of paint [4]. The signal was fed into a lockin amplifier whose reference was the oscillating strain of the sample.

The experiments were performed using tensile stress modulated at $10 \mathrm{~Hz}$. By variation of the modulation frequency the depth that is analysed can be modified (depth

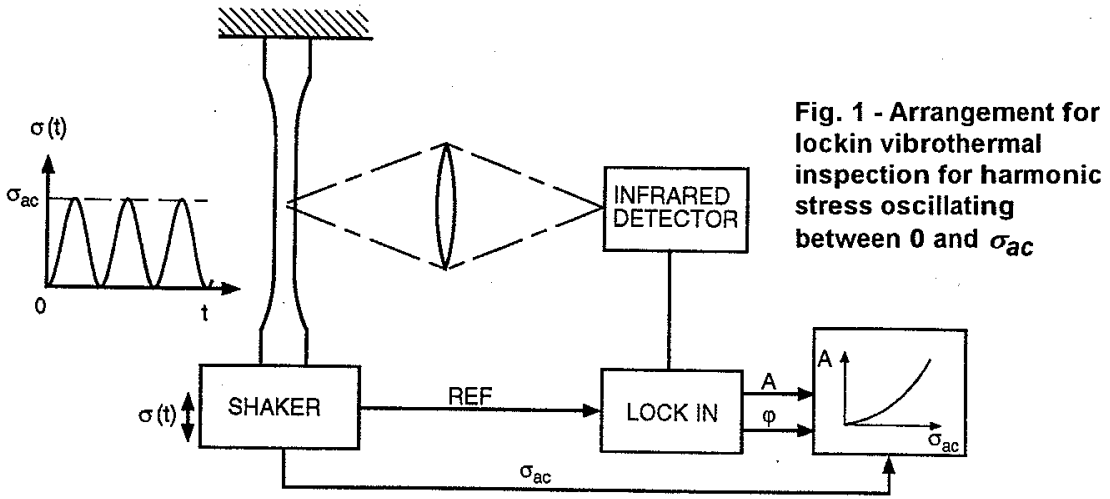


profiling). At $10 \mathrm{~Hz}$ the thermal diffusion length of polymers is less than $100 \mu \mathrm{m}$, hence only thermal waves from these near-surface areas of the sample contribute to the modulated infrared radiation emitted essentially from the surface. Infrared radiation from inside the sample can be neglected due to the high absorption in this spectral range.

Besides amplitude $A$ and phase $\varphi$ of the signal from the infrared detector we measured the dc-temperature (in a remote way using a thermography camera) at the surface and the strain. As variable parameters we used either the stress modulation amplitude or the stress offset at a constant small amplitude.

\section{Results}

\subsection{Amplitude dependence}

To illustrate the hysteresis effect, figure 2 shows stress-strain curves for various strain amplitudes up to $1.8 \%$ at $10 \mathrm{~Hz}$. The area of each of these curves is the part of elastic energy which is converted to volume heating. For this effect the amplitude $A$ of the infrared detector should be proportional to the second power of stress modulation $\sigma_{a c}$. However, the plot $\sqrt{A}$ as function of $\sigma_{a c}$ is not a straight line through the origin for the observed data (see figure 3 ). The reason cannot be the increase of average temperature: the deviation from a straight line is strongest at small stresses where also signal phase undergoes strong changes. The reason is the superposition of another effect, the thermoelastic effect where the temperature change is linear in stress [5-8]. In that case the infrared detector signal $S$ is given by:

$$
S=A\left(\sigma_{a c}\right) \exp \left[i \varphi\left(\sigma_{a c}\right)\right]=K_{t} \sigma_{a c}+K_{1} \sigma_{a c}^{2}
$$

where $K_{t}$ and $K_{1}$ (denoting the thermoelastic effect and the loss angle, respectively) are complex quantities. Therefore the strong change of signal phase indicates the change of the dominating mechanism. Above $10 \mathrm{MPa}$ or at strains above $0.2 \%$ the loss angle effect dominates. The influence of glass fibres is the reduction of strain at a given stress, hence a lower average temperature and a reduced temperature amplitude $A$ are observed. However, signal phase $\varphi$ is not much affected by the fibres besides indicating that the transition occurs now in the lower stress regime. In other experiments we found that the $\varphi$ curve is rather insensitive not only to fibres but also to humidity (even for polyamide kept in water for one week).

On this background it is surprising that $\varphi$ may be strongly affected by previous loading. For polyamide 6 with $30 \%$ glass fibres (PA 6-GF 30) the measurements were performed up to amplitudes of $1.5 \%$ (solid line figure 4 ) and then repeated after unloading. Up to strains of $30 \mathrm{MPA}$ (or strains of $0.5 \%$ ) all curves look the same except for the phase angle displaying a shift of about 8 degrees. Therefore this sensitivity of signal phase may be used to reveal the effects of previous loading already at a very low load level.

From work on the thermoelastic effect it is known that signal phase is sensitive to the application of paint to the material [4]. The same is true if we use the dissipated energy for thermal wave generation as shown in figure 5 where a scan was performed across polybutylene terephthalate partly provided with paint. The reason for this sensitivity is that from the bulk material to the monitored surface the thermal wave propagates across a layer that does not act as a thermal wave generator (or at least as a different thermal wave generator). Therefore we observe the influence of attenuated thermal wave transmission [9]. The paint itself may act as a thermal wave generator only if it adheres well enough to the substrate. In that case it is obvious that the pretreatment of the substrate prior to the application of paint will affect the magnitude of the observed thermal wave. To see how well the effect is applicable to monitor surface pretreatment 
http://dx.doi.org/10.21611/qirt.1992.024

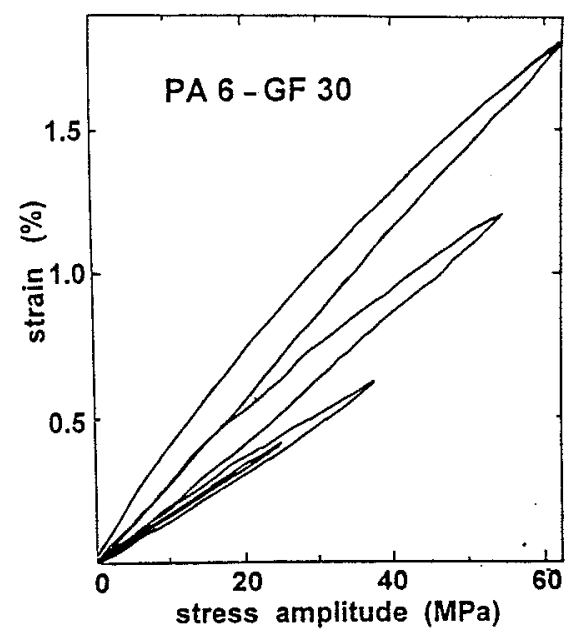

Fig. 2 - Hysteresis effect for various values of $\sigma_{a c}$

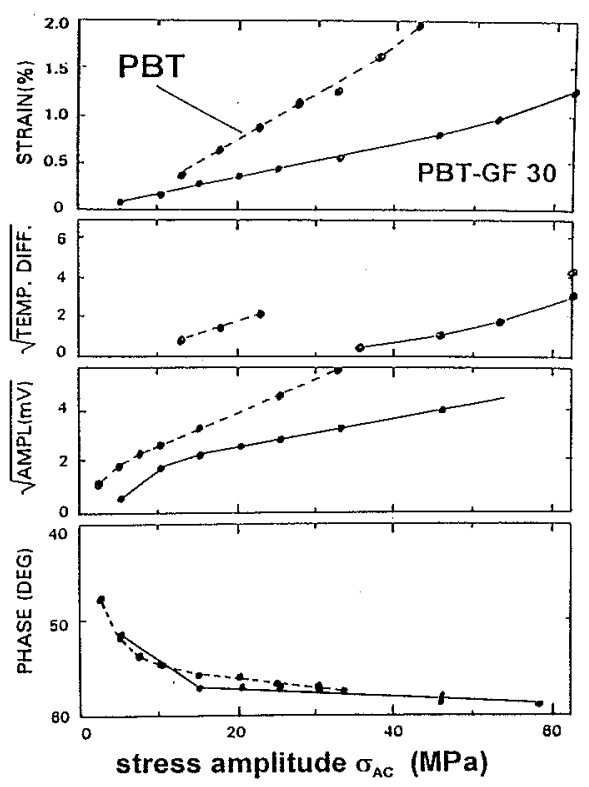

Fig. 3 - From top to bottom: strain amplitude, average temperature increase lobserved in conventional vibrothermography), amplitude and phase of lockin signal for PBT without (dashed line) and with $30 \%$ glass fibres (solid line)

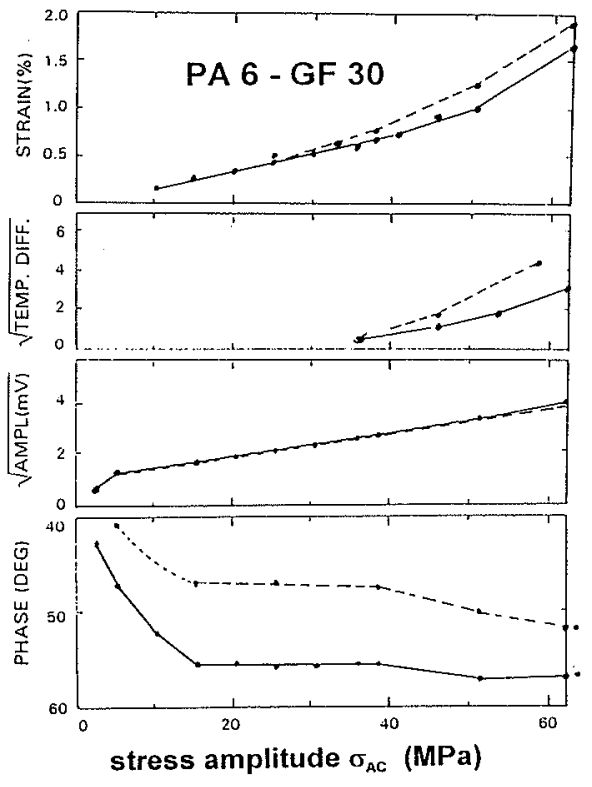

Fig. 4 - Effect of previous loading on PA 6 with glass fibres is strongest on signal phase (bottom) 


\section{http://dx.doi.org/10.21611/qirt.1992.024}
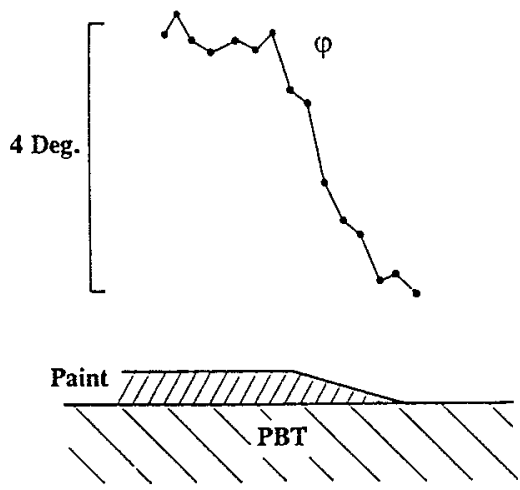

Fig 5 - Phase $\varphi$ observed during a onedimensional scan across PBTpartially painted

we measured the signal magnitude on nine samples. Paint and substrate were the same, but there were three different kinds of pretreatment. Accordingly we find three different groups of curves, each belonging to one kind of preparation (figure 6). Therefrom we conclude that lockin vibrothermal inspection may be useful to characterise the efficiency of methods applied to improve the adhesion of paint.
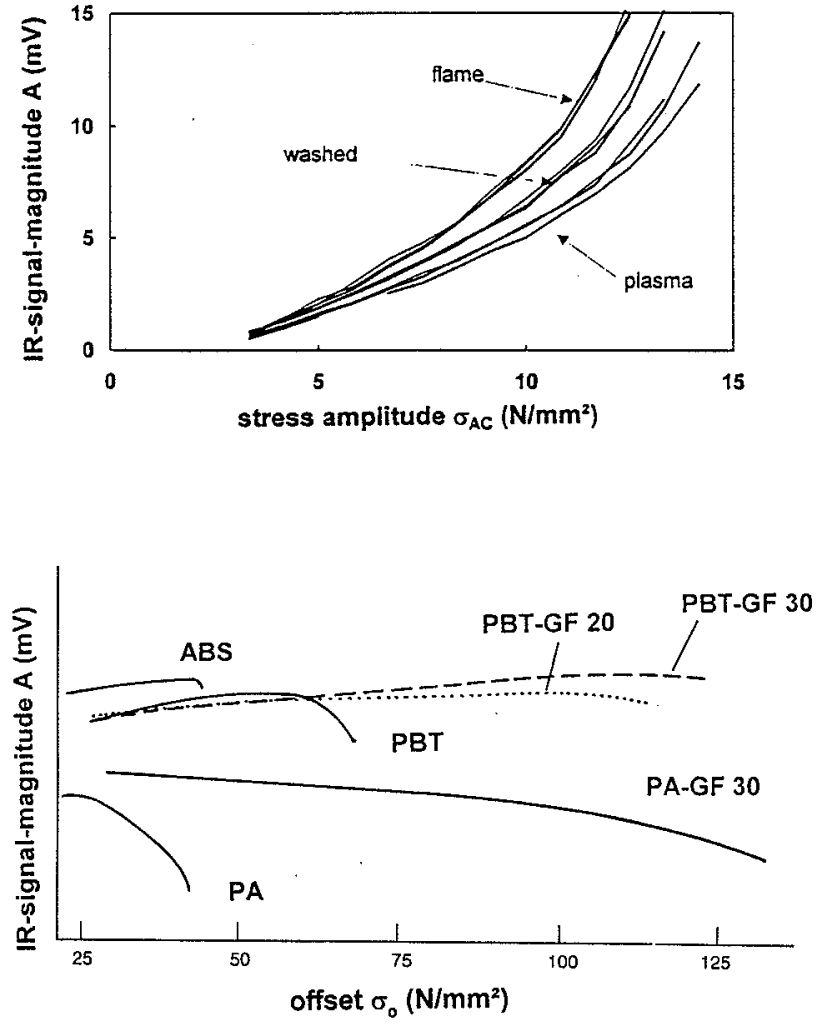

Fig. 6 - Influence of substrate pretreatment on signal of painted polymer (PP-EPDM)
Fig. 7 - Effect of stress offset $\sigma_{0}$ on various polymers with and without glass fibres 


\subsection{Offset dependence}

In these experiments a constant offset stress $\sigma_{0}$ was superposed to the oscillating load $\sigma_{\mathrm{ac}}$. While the measurements in paragraph 3.1 correspond to $\sigma_{0}=0$ and variable values of $\sigma_{a c}$, we measure now at a low constant amplitude $\sigma_{a c}$ while $\sigma_{0}$ is changed. Results for the magnitude curves $A\left(\sigma_{0}\right)$ of various materials are shown in figure 7 . The effect of fibres can roughly be understood from the strengthening effect. However, we do not obtain straight lines and constant values but a negative slope for PA and positive slopes for ABS and PBT. Also we find a decrease immediately before failure for all materials. Further analysis of these effects - which are specific for the material - or for its failure is of interest both for theory and applications.

\section{Conclusion}

Lockin vibroradiometry has been performed for various polymer materials. The measurements with variable stress amplitudes revealed the change from the thermoelastic effect to the loss angle effect as the dominating mechanism. Also they indicated the influence of preloading and coating. This kind of experiment is helpful not only for basic research to fit polymer model parameters, but also for applications in the field of non destructive testing since some effects occur already at low stress levels. The measurements at variable offset levels of stress may find their application in the prediction of material failure.

\section{REFERENCES}

[1] HENNEKE (E. G.), REIFSNIDER (K. L.) and STINCHCOMB (W.W.) Thermography - An NDI method for damage detection. Journal of Metals, Sept. 1979, p. 11-15.

[2] ROSENCWAIG (A), BUSSE (G) - High resolution photoacoustic thermal wave microscopy. Appl. Phys. Lett. 36, 1980, p. 725-727.

[3] BUSSE (G.), WU (D.) and KARPEN (W.). - Thermal wave imaging with phase sensitive modulated thermography. J. Appl. Phys., 71, 8, 1992, p. 3962-3965.

[4] Patent No. PCT/GB 79/00081 and DE 2952809 C2.

[5] WEBER (W) - Über die spezifische Wärmeleitung fester Körper, insbesondere der Metalle. Annalen der Physik u. Chemie 96 St 2 J, 1830, p. 177-213.

[6] THOMSON (W) - On the dynamical theory of heat. Trans. Roy.Soc. Edinburgh, 20,1853, p. $261-283$.

[7] WÖLFEL $(\mathrm{H})$ and FEICKERT (W) - Comparison of thermoelastic stress analysis and finite-element results. Proc. 9th Int. Conf. on Exp. Mech., Copenhagen, Denmark, August 1990, p. 748-757.

[8] FICKER (E) - Thermische Emissionsanalyse als Meßmethode in der Festkörpermechanik. Österr. Ing. und Arch. Zeitschr. 196 Nr. 7/8, 1991, p. 355-359.

[9] BUSSE (G) - Photothermal transmission probing of a metal. Infrared Physics 20, 1980, p. $419-422$. 